\title{
On the Well-posedness of a Class of Non-Autonomous SPDEs: An Operator-Theoretical Perspective
}

\author{
Rainer Picard ${ }^{1, *}$, Sascha Trostorff ${ }^{1, * *}$, and Marcus Waurick ${ }^{2, * * *}$ \\ ${ }^{1}$ TU Dresden, Fakultät Mathematik, Insitut für Analysis, 01062 Dresden, Germany \\ ${ }^{2}$ University of Strathclyde, Livingstone Tower, Department of Mathematics and Statistics, \\ G1 $1 \mathrm{XH}$, Glasgow, Scotland
}

Received XXXX, revised XXXX, accepted XXXX

Published online XXXX

Key words stochastic partial differential equations, evolutionary equations, stochastic equations of mathematical physics, weak solutions, non-autonomous equations, non-linear equations, differential inclusions.

MSC (2000) Primary 60H15, 35R60, Secondary 35Q99, 35F46

We further elaborate on the solvability of stochastic partial differential equations (SPDEs). We shall discuss non-autonomous partial differential equations with an abstract realization of the stochastic integral on the right-hand side. Our approach allows the treatment of equations with mixed type, where classical solution strategies fail to work. The approach extends prior observations in [Süß, A. \& Waurick, M. A Solution Theory for a General Class of SPDEs. Stochastics and Partial Differential Equations: Analysis and Computations, 2017, 5, 278-318], where the respective results were obtained for linear autonomous equations and (multiplicative) white noise.

Copyright line will be provided by the publisher

\section{Introduction}

In this article we discuss the well-posedness of and causality for a class of non-autonomous partial differential equations/inclusions perturbed with multiplicative noise. Our strategy is based on the rationale outlined in [15]. In this reference equations of the following type were discussed:

$$
\left(\partial_{0} M\left(\partial_{0}^{-1}\right)+A\right) u=\int_{0}^{\cdot} \sigma(u) d B(s),
$$

where $B$ is an appropriate (vector-valued) Brownian motion, $\sigma$ is a Lipschitz continuous mapping, $\partial_{0}$ is the time derivative, $M: z \mapsto M(z) \in L(H)$ is an analytic function that allows for defining $M\left(\partial_{0}^{-1}\right)$ by means of an appropriate functional calculus, and $A$ is a skew-selfadjoint operator in a Hilbert space $H$. It has been shown that many standard stochastic partial differential equations fit into the framework described by (1.1).

\footnotetext{
* E-mail: rainer.picard@tu-dresden.de

** E-mail: sascha.trostorff@tu-dresden.de

*** Corresponding author E-mail: marcus.waurick@strath.ac.uk, Phone: +44 1415483817
} 
In fact, the stochastic heat and wave equation with multiplicative noise are special cases of (1.1). In particular, it is also possible to formulate a version of Maxwell's equation with multiplicative noise.

In this article we will enlarge the admissible class of stochastic partial differential equations towards non-autonomous or even non-linear inclusions, which are subject to a stochastic perturbation of the right-hand side.

In a nutshell, the strategy outlined in [15], that is, a way to solving (1.1), is to find a Hilbert space that leads to

$$
u \mapsto\left(\partial_{0} M\left(\partial_{0}^{-1}\right)+A\right)^{-1} \int_{0}^{\cdot} \sigma(u) d B(s)
$$

being a strict contraction. In this exposition, an adapted result can be found in Theorem 5.11 (see also Theorem 5.12). In order to obtain the main result in [15], a key observation is that the operator $\left(\partial_{0} M\left(\partial_{0}^{-1}\right)+A\right)^{-1}$ is causal, which implies that in the fixed point iteration predictable processes are mapped to predictable processes, see also Theorem 5.8. The above mapping becomes a strict contraction as the Hilbert space setting is formulated in such a way that the Lipschitz constant of $u \mapsto \int_{0}^{*} \sigma(u) d B(s)$ can be made arbitrarily small, see Proposition 5.5 here.

In comparison to [15], we shall not elaborate so much on the classical notions of solving stochastic partial differential equations, but rather refer the reader instead to standard monographs such as $[13,14,18]$.

We shall describe the plan of this note next. After having exemplified typical applications of the rational developed in this manuscript, we establish - as a another key ingredient - the time derivative as a normal and continuously invertible operator in exponentially weighted Hilbert spaces. We recall the notion of evolutionary mappings and causality and draw some interconnections of these concepts. The basic fixed point theorem to be applied to stochastic partial differential equations can be found in Theorem 3.6.

Afterwards, in Section 4, we recall the essentials of the deterministic solution theory for non-autonomous equations. We shall also mention a non-linear variant of the solution theory at hand so that non-linear stochastic partial differential inclusions can like-wise be considered.

Section 5 is devoted to the discussion of stochastic evolutionary equations. In Section 5.1, we will set the stage for the probabilistic solution theory and rephrase the description of stochastic integration as outlined in [9]. The solution theory for non-autonomous stochastic evolutionary equations is provided in Section 5.2. More precisely, Theorems 5.11 and 5.12 are the main contributions of this manuscript substantially extending the main result of [15]. In Section 5.3, we conclude this article by providing some examples, which might be difficult - if not impossible - to treat with a more classical approach.

\section{A glimpse on some particular results}

In order to describe particular applications of the well-posedness results in this manuscript, we need to introduce some operators from vector analysis realised as certain unbounded operators in Hilbert spaces.

Throughout, let $\Omega \subseteq \mathbb{R}^{d}$ be an open set for some integer $d>0$. 
Definition 2.1 We define

$$
\begin{aligned}
& \operatorname{grad}: H_{0}^{1}(\Omega) \subseteq L^{2}(\Omega) \rightarrow L^{2}(\Omega)^{d}, \phi \mapsto\left(\partial_{i} \phi\right)_{i \in\{1, \ldots, d\}}, \\
& \text { Grad: } H_{0}^{1}(\Omega)^{d} \subseteq L^{2}(\Omega)^{d} \rightarrow L_{\mathrm{sym}}^{2}(\Omega)^{d \times d}, \Phi \mapsto \frac{1}{2}\left(\nabla \Phi+\nabla \Phi^{T}\right), \\
& \operatorname{curl}: H(\operatorname{curl}, \Omega) \subseteq L^{2}(\Omega)^{3} \rightarrow L^{2}(\Omega)^{3}, \Psi \mapsto\left(\sum_{j, k \in\{1,2,3\}} \varepsilon_{i j k} \partial_{j} \Psi_{k}\right)_{i \in\{1,2,3\}},
\end{aligned}
$$

where $H(\operatorname{curl}, \Omega)$ is the space of $L^{2}$-vector fields with distributional curl still being in $L^{2}$. The space $L_{\text {sym }}^{2}(\Omega)^{d \times d}$ denotes the set of symmetric $d$-by- $d$ matrices with entries in $L^{2}(\Omega)$.

We also put curl $:=$ curl $^{*}, \operatorname{div}:=-\operatorname{grad}^{*}$, and Div $:=-\operatorname{Grad}^{*}$. We note that for $\Omega$ with sufficiently smooth boundary belonging to the domain of curl corresponds to $H$ (curl, $\Omega$ )vector field with vanishing tangential component at the boundary. The definitions presented, however, do not require any regularity of the boundary.

In all the examples to come, we assume that $G$ is a separable Hilbert space and that $L \subseteq$ $L(G, H)$ satisfies the assumptions in 5.7 below for either of the choices

$$
H \in\left\{L^{2}(\Omega), L^{2}(\Omega)^{3} \oplus L^{2}(\Omega)^{3}, L^{2}(\Omega)^{d}\right\},
$$

which will be clear from the context. Moreover, we assume $\sigma: H \rightarrow L$ be Lipschitz continuous with $\sigma(0)=0$. In order to have a concrete example at hand, we shall choose $X=W$ to be the Wiener process with values in $G$ satisfying $W(t)=0$ for all $t \leq 0$. Note that in this case $\mathcal{F}$ is chosen to be the natural filtration of $W$ as in Example 5.4(a), which leads to $\alpha=\lambda \otimes \mathbb{P}\left(\lambda\right.$ denoting the Lebesgue measure on $\left.\mathbb{R}_{\geq 0}\right)$. For the definition of $\mathcal{I}_{\nu}^{W, \alpha}$ we refer to Section 5.1; also consult Corollary 5.10 for $\mathcal{I}^{W, \alpha} \circ \widetilde{\sigma}$.

Also we refer to Section 3 for a definition of $\partial_{0, \nu}$ and to Definition 5.2(c) for a definition of $L_{\nu, \mathrm{pr}}^{2}\left(\mathbb{R} ; L^{2}(\mathbb{R} ; H)\right)$.

\subsection{Standard linear examples}

We start out with a first order formulation of the heat equation. Let $a: \mathbb{R} \rightarrow L\left(L^{2}(\Omega)^{d}\right)$ be bounded, Lipschitz continuous with $a(t)=a(t)^{*} \geq c$ for all $t \in \mathbb{R}$ and some $c>0$; denote $b(t):=a(t)^{-1}$ and by $b^{\prime}$ the weak derivative of $b$.

Theorem 2.2 There exists $\nu>0$ such that for all $f \in L_{\nu, \mathrm{pr}}^{2}\left(\mathbb{R} ; L^{2}\left(\mathbb{P} ; L^{2}(\Omega)\right)\right)$ we find a uniquely determined $u_{f} \in L_{\nu, \mathrm{pr}}^{2}\left(\mathbb{R} ; L^{2}\left(\mathbb{P} ; L^{2}(\Omega)\right)\right.$ and $q_{f} \in L_{\nu, \mathrm{pr}}^{2}\left(\mathbb{R} ; L^{2}\left(\mathbb{P} ; L^{2}(\Omega)^{d}\right)\right.$ such that

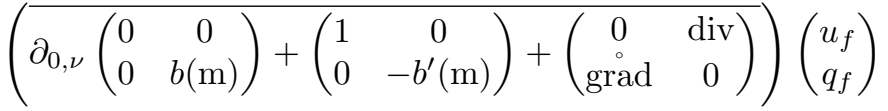

$$
\begin{aligned}
& =\left(\begin{array}{c}
\partial_{0, \nu}^{-1} f+\mathcal{I}^{W, \alpha} \circ \widetilde{\sigma}\left(u_{f}\right) \\
0
\end{array}\right) .
\end{aligned}
$$

Proof. This is a special case of Theorem 5.16 for $P=0, C=$ grad. 
We shall formally rewrite the equation satisfied by $\left(u_{f}, q_{f}\right)$ in Theorem 2.2 . We compute using the second equation,

$$
b(\mathrm{~m}) \partial_{0, \nu} q_{f}=\partial_{0, \nu} b(\mathrm{~m}) q_{f}-b^{\prime}(\mathrm{m}) q_{f}=-\stackrel{\operatorname{grad}}{\operatorname{rad}} u_{f}
$$

Hence,

$$
\partial_{0, \nu} q_{f}=-a(\mathrm{~m}) \operatorname{grad} u_{f}
$$

This, in turn, leads to, using the first equation,

$$
\partial_{0, \nu}^{-1} f+\mathcal{I}^{W, \alpha} \circ \widetilde{\sigma}\left(u_{f}\right)=u_{f}+\operatorname{div} q_{f}=u_{f}-\partial_{0, \nu}^{-1} \operatorname{div} a(\mathrm{~m}) \operatorname{grad} u_{f},
$$

or,

$$
\partial_{0, \nu} u_{f}-\operatorname{div} a(\mathrm{~m}) \operatorname{grad} u_{f}=f+\partial_{0, \nu} \mathcal{I}^{W, \alpha} \circ \widetilde{\sigma}\left(u_{f}\right),
$$

which is the stochastic heat equation.

Quite similarly, one can deal with the stochastic wave equation. We shall, however, discuss a different hyperbolic type example next - the stochastic Maxwell's equations. For this, we let $\varepsilon, \mu, \eta: \mathbb{R} \rightarrow L\left(L^{2}(\Omega)^{3}\right)$ be bounded, Lipschitz continuous with $\varepsilon(t)=\varepsilon(t)^{*}, \mu(t)=$ $\mu(t)^{*} \geq c$ for all $t \in \mathbb{R}$ and some $c \geq 0$. In this case $\varepsilon, \mu, \eta$ describe the dielectricity, the magnetic permeability, and the electric conductivity, respectively.

Theorem 2.3 There exists $\nu>0$ such that for all $(J, K) \in L_{\nu, \mathrm{pr}}^{2}\left(\mathbb{R} ; L^{2}\left(\mathbb{P} ; L^{2}(\Omega)^{3} \oplus\right.\right.$ $\left.\left.L^{2}(\Omega)^{3}\right)\right)$ there exists uniquely determined $(E, H) \in L_{\nu, \mathrm{pr}}^{2}\left(\mathbb{R} ; L^{2}\left(\mathbb{P} ; L^{2}(\Omega)^{3} \oplus L^{2}(\Omega)^{3}\right)\right)$ such that

$$
\begin{array}{r}
\left(\begin{array}{c}
\left.\partial_{0, \nu}\left(\begin{array}{cc}
\varepsilon(\mathrm{m}) & 0 \\
0 & \mu(\mathrm{m})
\end{array}\right)+\left(\begin{array}{cc}
\eta(\mathrm{m}) & 0 \\
0 & 0
\end{array}\right)+\left(\begin{array}{cc}
0 & -\mathrm{curl} \\
0 & 0 \\
\operatorname{curl} & 0
\end{array}\right)\right) \\
=\left(\begin{array}{c}
E \\
H
\end{array}\right) \\
K
\end{array}\right)+\mathcal{I}^{W, \alpha} \circ \tilde{\sigma}((E, H)) .
\end{array}
$$

Proof. The result follows upon applying Theorem 5.11, which in turn prerequisites the validity of Assumption 4.1 under the setting:

$$
\begin{array}{lll}
H=L^{2}(\Omega)^{3} \oplus L^{2}(\Omega)^{3}, & & A=\left(\begin{array}{cc}
0 & - \text { curl } \\
\text { curl } & 0
\end{array}\right), \\
\mathcal{M}=\left(\begin{array}{cc}
\varepsilon(\mathrm{m}) & 0 \\
0 & \mu(\mathrm{m})
\end{array}\right), & \mathcal{M}^{\prime}=\left(\begin{array}{cc}
\varepsilon^{\prime}(\mathrm{m}) & 0 \\
0 & \mu^{\prime}(\mathrm{m})
\end{array}\right) \\
\mathcal{N}=\left(\begin{array}{cc}
\eta(\mathrm{m}) & 0 \\
0 & 0
\end{array}\right) . &
\end{array}
$$

By Lemma 5.13 applied to $C=-\operatorname{corl}, A$ is m-accretive. It is also elementary to see that $\mathcal{M}$, $\mathcal{M}^{\prime}$ and $\mathcal{N}$ satisfy the positive definiteness conditions, we refer to Lemma 5.15 for a similar argument. This concludes the proof. 


\subsection{A nonlinear example}

We conclude this examples section with a stochastic variant of the equations of viscoplasticity with internal variables. We refer to $[1,17]$ for a deterministic model.

In the stochastic setting discussed here, we shall assume that the equations governing the displacement field $u$ are stochastically perturbed and that the nonlinearity is slightly different from the equations discussed in [1,17]. Let $N \in \mathbb{N}$. The system to be studied reads

$$
\begin{aligned}
\partial_{0, \nu}^{2} R u-\operatorname{Div} T & =f+\partial_{0, \nu} \mathcal{I}^{W, \alpha} \circ \widetilde{\sigma}(u), \\
T & =D(\operatorname{Grad} u-B z), \\
\left(B^{*} \partial_{0, \nu}^{-1} T-L \partial_{0, \nu}^{-1} z, z\right) & \in g
\end{aligned}
$$

where $R: \mathbb{R} \rightarrow L\left(L^{2}(\Omega)^{d}\right), D: \mathbb{R} \rightarrow L\left(L_{\text {sym }}^{2}(\Omega)^{d \times d}\right), L: \mathbb{R} \rightarrow L\left(L^{2}(\Omega)^{N}\right)$ are bounded, Lipschitz continuous, $R(t)=R(t)^{*}, L(t)=L(t)^{*}, D(t)=D(t)^{*}$ with $R(t) \geq c, D(t) \geq c$ and $L(t) \geq c$ for all $t \in \mathbb{R}$ and some $c>0$. D is the elasticity tensor, $f$ is a given volume force, $B \in L\left(L^{2}(\Omega)^{N}, L_{\text {sym }}^{2}(\Omega)^{d \times d}\right)$ describes the inelastic part $e_{p}=B z$ of the strain tensor $e=\operatorname{Grad} u ; g \subseteq L^{2}(\Omega)^{N} \oplus L^{2}(\Omega)^{N}$ is a maximal monotone relation with $(0,0) \in g$. The unknowns of the above model are the displacement $u$, the stress tensor $T$, and the vector of internal variables $z$, where the latter assumes values in $L^{2}(\Omega)^{N}$.

We reformulate the system (2.1)-(2.3). For this, we introduce

$$
\hat{T}:=\partial_{0, \nu}^{-1} T \text { and } w:=B^{*} \hat{T}-L(\mathrm{~m}) \partial_{0, \nu}^{-1} z .
$$

Then (2.1) reads

$$
\partial_{0, \nu} R(\mathrm{~m}) u-\operatorname{Div} \hat{T}=\partial_{0, \nu}^{-1} f+\mathcal{I}^{W, \alpha} \circ \widetilde{\sigma}(u) .
$$

Furthermore, (2.3) becomes

$$
\left(w, \partial_{0, \nu} L^{-1}(\mathrm{~m})\left(B^{*} \hat{T}-w\right)\right) \in g
$$

and (2.2) yields

$$
\partial_{0, \nu} D^{-1}(\mathrm{~m}) \hat{T}-\left(D^{-1}\right)^{\prime}(\mathrm{m}) \hat{T}=D^{-1}(\mathrm{~m}) \partial_{0, \nu} \hat{T}=\operatorname{Grad} u-\partial_{0, \nu} B L^{-1}(\mathrm{~m})\left(B^{*} \hat{T}-w\right) .
$$

Altogether, we obtain

$$
\left(\left(\begin{array}{c}
u \\
w \\
\hat{T}
\end{array}\right),\left(\begin{array}{c}
\partial_{0, \nu}^{-1} f+\mathcal{I}^{W, \alpha} \circ \widetilde{\sigma}(u) \\
0 \\
0
\end{array}\right)\right) \in \partial_{0, \nu} \mathcal{M}+\mathcal{N}+A .
$$


with

$$
\begin{aligned}
\mathcal{M} & =\left(\begin{array}{ccc}
R(\mathrm{~m}) & 0 & 0 \\
0 & L^{-1}(\mathrm{~m}) & -L^{-1}(\mathrm{~m}) B^{*} \\
0 & -B L^{-1}(\mathrm{~m}) & D^{-1}(\mathrm{~m})+B L^{-1}(\mathrm{~m}) B^{*}
\end{array}\right) \\
\mathcal{N} & =\left(\begin{array}{ccc}
0 & 0 & 0 \\
0 & 0 & 0 \\
0 & 0 & -\left(D^{-1}\right)^{\prime}(\mathrm{m})
\end{array}\right) \text { and } \\
A & =\left(\begin{array}{ccc}
0 & 0 & - \text { Div } \\
0 & g & 0 \\
- \text { Grad } & 0 & 0
\end{array}\right) .
\end{aligned}
$$

By applying a symmetric Gauss-step, it is not difficult to show that the operators $\mathcal{M}, \mathcal{N}$ satisfy the assumptions stated in Theorem 5.12 with $H=L^{2}(\Omega)^{d} \oplus L^{2}(\Omega)^{N} \oplus L_{\text {sym }}^{2}(\Omega)^{d \times d}$. Moreover, note that by [17, p. 64], also the relation $A$ satisfies the assumptions in Theorem 5.12. Thus, we have shown the following result:

Theorem 2.4 Let $\mathcal{M}, \mathcal{N}$ and $A$ as above. Then there exists $\nu>0$ such that for all $f \in L_{\nu, \mathrm{pr}}^{2}\left(\mathbb{R} ; L^{2}\left(\mathbb{P} ; L^{2}(\Omega)^{d}\right)\right)$ there exists a unique $(u, w, \hat{T}) \in L_{\nu, \mathrm{pr}}^{2}\left(\mathbb{R} ; L^{2}\left(\mathbb{P} ; L^{2}(\Omega)^{d} \oplus\right.\right.$ $\left.\left.L^{2}(\Omega)^{N} \oplus L_{\text {sym }}^{2}(\Omega)^{d \times d}\right)\right)$ satisfying

$$
\overline{\partial_{0, \nu} \mathcal{M}+\mathcal{N}+A} \ni\left((u, w, \hat{T}),\left(\partial_{0, \nu}^{-1} f+\mathcal{I}^{W, \alpha} \circ \widetilde{\sigma}(u), 0,0\right)\right) .
$$

We shall now develop the theory in order to properly justify the above results.

\section{The time derivative and evolutionary mappings}

Let $H$ be a Banach space. For $\nu \in \mathbb{R}$ we define

$$
L_{\nu}^{2}(\mathbb{R} ; H):=\left\{f \in L_{\mathrm{loc}}^{2}(\mathbb{R} ; H) ; \int_{\mathbb{R}}|f(t)|_{H}^{2} \exp (-2 \nu t) \mathrm{d} t<\infty\right\}
$$

endowed with the obvious norm. It is easy to see that $L_{\nu}^{2}(\mathbb{R} ; H)$ is a Banach space, as well. Specializing to $H$ being a Hilbert space, we denote by $H_{\nu}^{1}(\mathbb{R} ; H)$ the Sobolev space of once weakly differentiable functions with derivative in $L_{\nu}^{2}(\mathbb{R} ; H)$. We obtain (see [7, Section 2]), that

$$
\begin{aligned}
\partial_{0, \nu}: H_{\nu}^{1}(\mathbb{R} ; H) \subseteq L_{\nu}^{2}(\mathbb{R} ; H) & \rightarrow L_{\nu}^{2}(\mathbb{R} ; H) \\
f & \mapsto f^{\prime}
\end{aligned}
$$

is a densely defined, closed and normal linear operator. Moreover, we have $\partial_{0, \nu}^{*}=-\partial_{0, \nu}+$ $2 \nu$. In applications to be discussed later on, $\partial_{0, \nu}$ will be our realization of the time derivative for $\nu>0$ 'large enough'. Note that for $\nu>0$, we obtain that $\partial_{0, \nu}$ is continuously invertible with

$$
\partial_{0, \nu}^{-1} f(t)=\int_{-\infty}^{t} f(\tau) \mathrm{d} \tau
$$


where the integral is well-defined for all $f \in L_{\nu}^{2}(\mathbb{R} ; H)$ in the Bochner sense and we have $\left\|\partial_{0, \nu}^{-1}\right\|=1 / \nu$, see also [7, Corollary 2.5].

For the treatment of evolutionary equations with non-autonomous coefficients, we will need the notion of evolutionary mappings. In fact, also in the discussion of stochastic partial differential equations, this notion proved useful for the abstract description of the stochastic integral.

Definition 3.1 Let $H, G$ be Banach spaces, $\nu>0$. Let

$$
F: \operatorname{dom}(F) \subseteq \bigcap_{\mu \geq \nu} L_{\mu}^{2}(\mathbb{R} ; H) \rightarrow \bigcap_{\mu \geq \nu} L_{\mu}^{2}(\mathbb{R} ; G),
$$

where $\operatorname{dom}(F)$ is supposed to be a vector space. We call $F$ evolutionary (at $\nu$ ), if for all $\mu \geq \nu, F$ satisfies the following properties

(i) $F$ is Lipschitz continuous as a mapping

$$
F_{0, \mu}: \operatorname{dom}(F) \subseteq L_{\mu}^{2}(\mathbb{R} ; H) \rightarrow L_{\mu}^{2}(\mathbb{R} ; G), \phi \mapsto F(\phi),
$$

(ii) $\|F\|_{\text {ev,Lip }}:=\lim \sup _{\mu \rightarrow \infty}\left\|F_{\mu}\right\|_{\text {Lip }}<\infty$, with $F_{\mu}:=\overline{F_{0, \mu}}$ denoting the Lipschitz continuous extension of $F$.

The non-negative number $\|F\|_{\mathrm{ev}, \mathrm{Lip}}$ is called the the eventual Lipschitz constant of $F$. We denote

$$
L_{\mathrm{ev}, \nu}(H, G):=\{F ; F \text { evolutionary at } \nu\}, \quad L_{\mathrm{ev}, \nu}(H):=L_{\mathrm{ev}, \nu}(H, H) .
$$

If, in addition, $F_{\mu}$ leaves $\operatorname{dom}\left(F_{\mu}\right)=\overline{\operatorname{dom}(F)}^{L_{\mu}^{2}}$ invariant $(\mu \geq \nu)$, then we call $F$ invariant evolutionary $(a t \nu)$. A mapping $F$, which is evolutionary at $\nu$, is called densely defined, if, for all $\mu \geq \nu, \operatorname{dom}(F) \subseteq L_{\mu}^{2}(\mathbb{R} ; H)$ is dense.

Next, we introduce the concept of causality, as it has been introduced in [19] as a particular concept for (nonlinear) mappings in Banach spaces. In the applications to follow, we will mainly focus on Lipschitz continuous Hilbert space valued mappings, see also [21, Definition 2.2.2 and Remark 2.2.3].

Definition 3.2 (a) Let $H$ be a Banach space. A family $R=\left(R_{t}\right)_{t \in \mathbb{R}}$ is called resolution of the identity (in $H$ ), if for all $t \in \mathbb{R}$

$$
R_{t}=R_{t}^{2} \in L(H) \text { and } 2 \lim _{t \rightarrow \pm \infty} R_{t}=1 \pm 1
$$

where the limit is in the strong operator topology of $L(H)$. The pair $(H, R)$ is called resolution space.

(b) Let $(H, R),(K, Q)$ be resolution spaces and let $F: \operatorname{dom}(F) \subseteq H \rightarrow K, D \subseteq K^{\prime}$. We call $F$ causal on $D$, if for all $r>0, t \in \mathbb{R}, \phi \in D$, the mapping

$$
\left(B_{F}(0, r),\left|R_{t}(\cdot-\cdot)\right|\right) \rightarrow\left(K,\left|\left\langle Q_{t}(\cdot-\cdot), \phi\right\rangle\right|\right), x \mapsto F(x)
$$

is Lipschitz continuous, where $B_{F}(0, r):=\left\{x \in \operatorname{dom}(F) ;|x|^{2}+|F(x)|^{2}<r\right\}$; if $D=K^{\prime}$, we call $F$ causal. 
Proposition 3.3 ( [19, Theorem 1.7] and [21, Theorem 2.2.4]) Let $(H, R),(K, Q)$ be resolution spaces, $F: \operatorname{dom}(F) \subseteq H \rightarrow K$ densely defined and Lipschitz continuous, $D \subseteq$ $K^{\prime}$ separating for $K$. Then the following conditions are equivalent:

(i) F is causal on D,

(ii) $\bar{F}$ is causal,

(iii) for all $t \in \mathbb{R}$,

$$
\left(\operatorname{dom}(F),\left|R_{t}(\cdot-\cdot)\right| \rightarrow\left(K,\left|Q_{t}(\cdot-\cdot)\right|\right), x \mapsto F(x)\right.
$$

is Lipschitz continuous.

(iv) for all $t \in \mathbb{R}$, we have $Q_{t} \circ \bar{F}=Q_{t} \circ \bar{F} \circ R_{t}$.

Proof. The implication (ii) $\Rightarrow$ (i) is trivial, both the implications (iii) $\Rightarrow$ (ii) and (iv) $\Rightarrow$ (iii) are easy to obtain. Thus, it suffices to prove that (i) is sufficient for (iv). For this, let $t \in \mathbb{R}$ and $\phi \in D$. For $\psi \in \operatorname{dom}(F)$ we find $\psi_{n} \in \operatorname{dom}(F)$ such that $\psi_{n} \rightarrow R_{t} \psi$ in $H$ as $n \rightarrow \infty$. By the boundedness of $\left(\psi_{n}\right)_{n}$ in $H$, and by causality of $F$ on $D$, we find $C \geq 0$ such that for all $n \in \mathbb{N}$, we obtain

$$
\left|\left\langle Q_{t} F(\psi)-Q_{t} F\left(\psi_{n}\right), \phi\right\rangle\right| \leq C\left|R_{t} \psi-R_{t} \psi_{n}\right| .
$$

Letting $n \rightarrow \infty$ in the latter inequality and using that $R_{t}^{2}=R_{t}$, we deduce that

$$
\left|\left\langle Q_{t} F(\psi)-Q_{t} \bar{F}\left(R_{t} \psi\right), \phi\right\rangle\right| \leq C\left|R_{t} \psi-R_{t}^{2} \psi\right|=0 .
$$

Thus, since $D$ is separating for $K$, we infer

$$
Q_{t} F(\psi)=Q_{t} \bar{F}\left(R_{t} \psi\right) \quad(\psi \in \operatorname{dom}(F)) .
$$

By continuity, we obtain $Q_{t} \circ \bar{F}=Q_{t} \circ \bar{F} \circ R_{t}$.

We recall a variant of [15, Lemma 2.13]. As the assumptions vary slightly from the ones used in [15, Lemma 2.13], we carry out the proof.

Lemma 3.4 Let $F$ be evolutionary at $\nu>0$. Assume that $\operatorname{dom}(F) \cap \operatorname{dom}\left(F \chi_{(-\infty, a]}\right)$ is dense in $\operatorname{dom}(F)$ with respect to the $L_{\mu}^{2}(\mathbb{R} ; H)$-norm for all $a \in \mathbb{R}$ and $\mu \geq \nu$. Then $\left.F_{\eta}\right|_{\operatorname{dom}\left(F_{\eta}\right) \cap \operatorname{dom}\left(F_{\mu}\right)}=\left.F_{\mu}\right|_{\operatorname{dom}\left(F_{\eta}\right) \cap \operatorname{dom}\left(F_{\mu}\right)}$ for all $\eta \geq \mu \geq \nu$.

Proof. Let $\phi \in \operatorname{dom}\left(F_{\eta}\right) \cap \operatorname{dom}\left(F_{\mu}\right)$. By assumption, we can choose a sequence $\left(\phi_{n}\right)_{n}$ in $\operatorname{dom}(F) \cap \operatorname{dom}\left(F \chi_{(-\infty, a]}\right)$ such that $\phi_{n} \rightarrow \phi$ in $L_{\mu}^{2}(\mathbb{R} ; H)$ as $n \rightarrow \infty$. Moreover, we deduce that $\operatorname{dom}(F) \ni \chi_{(-\infty, a]} \phi_{n} \rightarrow \chi_{(-\infty, a]} \phi$ in both $L_{\eta}^{2}(\mathbb{R} ; H)$ and $L_{\mu}^{2}(\mathbb{R} ; H)$ as $n \rightarrow \infty$. In particular, we obtain $\chi_{(-\infty, a]} \phi \in \operatorname{dom}\left(F_{\mu}\right) \cap \operatorname{dom}\left(F_{\eta}\right)$ and

$$
\begin{aligned}
F_{\mu}\left(\chi_{(-\infty, a]} \phi\right) & =\lim _{n \rightarrow \infty} F_{\mu}\left(\chi_{(-\infty, a]} \phi_{n}\right) \\
& =\lim _{n \rightarrow \infty} F\left(\chi_{(-\infty, a]} \phi_{n}\right) \\
& =\lim _{n \rightarrow \infty} F_{\eta}\left(\chi_{(-\infty, a]} \phi_{n}\right)=F_{\eta}\left(\chi_{(-\infty, a]} \phi\right) .
\end{aligned}
$$

Next, we note that $\chi_{(-\infty, a]} \phi \rightarrow \phi$ as $a \rightarrow \infty$ in $L_{\eta}^{2}(\mathbb{R} ; H)$ and $L_{\mu}^{2}(\mathbb{R} ; H)$ since $\phi \in$ $L_{\eta}^{2}(\mathbb{R} ; H) \cap L_{\mu}^{2}(\mathbb{R} ; H)$. Hence, $F_{\mu}(\phi)=F_{\eta}(\phi)$. 
We shall further point out another consequence of evolutionarity and the condition on the domain in the previous result. In fact, this is a combination of the arguments used for [7, Theorem 4.5] and [21, Remark 2.1.5]. For this, from now on and throughout the whole manuscript, we shall use $R_{t}=Q_{t}=\chi_{(-\infty, t]}$ as the standard resolution of the identity, and thus $\left(L_{\nu}^{2}(\mathbb{R} ; H),\left(\chi_{(-\infty, t]}\right)_{t}\right)$ as resolution space.

Lemma 3.5 Let $F$ be evolutionary at $\nu>0$. Assume that $\operatorname{dom}(F) \cap \operatorname{dom}\left(F \chi_{(-\infty, a]}\right)$ is dense in $\operatorname{dom}(F)$ with respect to the $L_{\mu}^{2}(\mathbb{R} ; H)$-norm for all $a \in \mathbb{R}$ and $\mu \geq \nu$. Then $F_{\mu}$ is causal for all $\mu \geq \nu$.

Proof. Let $\mu \geq \nu$. We apply Proposition 3.3 and prove $Q_{t} \circ F=Q_{t} \circ F \circ Q_{t}$ for all $t \in \mathbb{R}$. Note that this implies (iv) in Proposition 3.3 as both the left- and the right-hand side are densely defined in $\operatorname{dom}\left(F_{\mu}\right)$. So, let $t \in \mathbb{R}, \phi \in \stackrel{\circ}{C}_{\infty}\left(\mathbb{R} ; G^{\prime}\right)$ and $\psi \in \operatorname{dom}(F) \cap$ $\operatorname{dom}\left(F \chi_{(-\infty, t]}\right)$. We compute for $\eta \geq \mu$

$$
\begin{aligned}
& \left|\left\langle Q_{t}\left(F(\psi)-F\left(Q_{t} \psi\right)\right), \phi\right\rangle_{L^{2}(\mathbb{R} ; G), L^{2}\left(\mathbb{R} ; G^{\prime}\right)}\right| \\
& =\left|\left\langle\left(F(\psi)-F\left(Q_{t} \psi\right)\right), Q_{t} \phi\right\rangle_{L^{2}(\mathbb{R} ; G), L^{2}\left(\mathbb{R} ; G^{\prime}\right)}\right| \\
& \leq\left\|F_{\eta}\right\|_{\text {Lip }}\left\|\psi-Q_{t} \psi\right\|_{L_{\eta}^{2}}\left\|Q_{t} \phi\right\|_{L^{2}} \exp (\eta t) .
\end{aligned}
$$

We compute further

$$
\begin{aligned}
\left\|\psi-Q_{t} \psi\right\|_{L_{\eta}^{2}}^{2} \exp (2 \eta t) & =\int_{\mathbb{R}}\left|\psi(s)\left(1-\chi_{(-\infty, t]}(s)\right)\right| \exp (-2 \eta(s-t)) \mathrm{d} s \\
& =\int_{\mathbb{R}}\left|\psi(s+t)\left(1-\chi_{(-\infty, t]}(s+t)\right)\right| \exp (-2 \eta s) \mathrm{d} s \\
& =\int_{0}^{\infty}|\psi(s+t)| \exp (-2 \eta s) \mathrm{d} s \rightarrow 0 \quad(\eta \rightarrow 0),
\end{aligned}
$$

and thus, we deduce that $Q_{t}\left(F(\psi)-F\left(Q_{t} \psi\right)\right)=0$, as desired.

We conclude this section with a perturbation result, which we need for a solution theory for non-autonomous stochastic partial differential equations.

Theorem 3.6 (see also [15, Corollary 2.15]) Let $H$ be a Banach space, $\nu>0, S, F \in$ $L_{\mathrm{ev}, \nu}(H), F$ invariant evolutionary. Let $S$ be densely defined, $\|S\|_{\mathrm{ev}, \mathrm{Lip}}\|F\|_{\mathrm{ev}, \mathrm{Lip}}<1$, and $S_{\mu}\left[\operatorname{dom}\left(F_{\mu}\right)\right] \subseteq \operatorname{dom}\left(F_{\mu}\right)$ for all $\mu \geq \nu$. Then for all $f \in \operatorname{dom}\left(F_{\mu}\right)$ the mapping

$$
\begin{aligned}
\Phi_{\mu}(f): \operatorname{dom}\left(F_{\mu}\right) & \rightarrow \operatorname{dom}\left(F_{\mu}\right) \\
u & \mapsto S_{\mu} f+S_{\mu}\left(F_{\mu}(u)\right)
\end{aligned}
$$

admits a unique fixed point $u_{f}$ as long as $\mu \geq \nu$ is large enough, that is, a unique solution $u_{f}$ of the problem

$$
u_{f}-S_{\mu}\left(F_{\mu}\left(u_{f}\right)\right)=S_{\mu}(f) .
$$

The mapping $f \mapsto u_{f}$ is evolutionary. If $S$ and $F$ are causal, then so is $f \mapsto u_{f}$. If $\operatorname{dom}(F) \cap$ $\operatorname{dom}\left(F \chi_{(-\infty, a]}\right)$ is dense in $\operatorname{dom}(F)$ with respect to $L_{\mu}^{2}(\mathbb{R} ; H)$ for all sufficiently large $\mu$, then $f \mapsto u_{f}$ does not depend on $\mu$ in the sense of Lemma 3.4. 
Proof. Let $\mu \geq \nu$ such that $\left\|S_{\mu}\right\|_{\text {Lip }}\left\|F_{\mu}\right\|_{\text {Lip }}<1$. Then it is easy to see that $\Phi_{\mu}(f)$ defines a strict contraction. By standard a posteriori estimates, we deduce that we find $C \geq 0$ such that $\left\|f \mapsto u_{f}\right\|_{\text {Lip }} \leq 1 /\left(1-\|S\|_{\text {ev,Lip }}\|F\|_{\text {ev,Lip }}\right)+C$.

It remains to prove causality of the fixed point mapping. For this it suffices to observe that $\Phi_{\mu}(f)$ is causal. This, however, follows from the fact that composition of causal mappings is still causal.

The independence of $\mu$ is a consequence of Lemma 3.4.

In applications, the mapping $S_{\mu}$ will be the solution operator of an abstract deterministic partial differential equation and thus, the solution $u_{f}$ in (3.1) turns out to be the solution of this deterministic PDE perturbed by an additional mapping $F_{\mu}$, which will be our stochastic integral operator.

\section{The deterministic solution theory}

In this section we will review the solution theory for a class of (non-autonomous) linear partial differential equations which has its roots in the autonomous version presented in [11]. Later on, this has been generalized to non-autonomous or non-linear equations, see e.g. [12, 16, 17, 20]. To keep this article conveniently self-contained, we shall summarize the well-posedness theorem outlined in [21, Theorem 3.4.6]. However, we will also present the main results of [17], in order to obtain a non-linear variant for stochastic partial differential equations.

The main hypothesis for the linear case is presented next.

Assumption 4.1 ( [21, Hypothesis 3.4.4]) Let $H$ be a Hilbert space, $\nu>0, \mathcal{M}, \mathcal{M}^{\prime}, \mathcal{N} \in$ $L_{\text {ev }, \nu}(H)$. Assume that $\stackrel{\circ}{C}_{\infty}(\mathbb{R} ; H) \subseteq \operatorname{dom}(\mathcal{M}) \cap \operatorname{dom}\left(\mathcal{M}^{\prime}\right) \cap \operatorname{dom}(\mathcal{N})$. Let $A: \operatorname{dom}(A) \subseteq$ $H \rightarrow H$ be densely defined and $\mathrm{m}$-accretive. Assume that

$$
\begin{aligned}
& \forall \mu \geq \nu: \mathcal{M} \partial_{0, \mu} \subseteq \partial_{0, \mu} \mathcal{M}_{\mu}-\mathcal{M}_{\mu}^{\prime}, \\
& \exists c>0 \forall \mu \geq \nu, t \in \mathbb{R}: \Re\left\langle Q_{t}\left(\partial_{0, \mu} \mathcal{M}+\mathcal{N}\right) \phi, \phi\right\rangle_{0, \mu} \geq c\left\langle\phi, Q_{t} \phi\right\rangle_{0, \mu} \\
& \qquad\left(\phi \in \stackrel{C}{C}_{\infty}(\mathbb{R} ; H)\right) .
\end{aligned}
$$

With the latter set of assumptions, we can show the following well-posedness theorem covering a large class of linear non-autonomous evolutionary equations:

Theorem 4.2 ( [21, Theorem 3.4.6]) Impose Assumption 4.1. Then the operator

$$
\mathcal{B}:=\partial_{0, \nu} \mathcal{M}+\mathcal{N}+A
$$

is densely defined and closable. Moreover, its closure is onto and continuously invertible in $L_{\mu}^{2}(\mathbb{R} ; H)$ for all $\mu \geq \nu$. Furthermore, $\mathcal{S}:=\mathcal{B}^{-1}$ is evolutionary at $\nu$, densely defined and causal.

Lemma 4.3 Let $G$ be a Hilbert space, $B: \operatorname{dom}(B) \subseteq G \rightarrow G$ a densely defined linear operator. Assume there exists $c>0$ with the property that

$$
\Re\langle B \phi, \phi\rangle \geq c\langle\phi, \phi\rangle,
$$

as well as

$$
\Re\left\langle B^{*} \psi, \psi\right\rangle \geq c\langle\psi, \psi\rangle,
$$


for all $\phi \in \operatorname{dom}(B)$ and $\psi \in \operatorname{dom}\left(B^{*}\right)$. Then $B$ is closable and $\bar{B}^{-1}$ exists as an element of $L(G)$, the space of bounded linear operators on $G$ and $\left\|\bar{B}^{-1}\right\| \leq 1 / c$.

Proof. Before we come to the proof of the assertion, we need some preparations. Note that for $\lambda>0$ the operator $1+\lambda B$ is one-to-one by (4.1). Moreover, by (4.2) we infer that its adjoint $1+\lambda B^{*}$ is one-to-one, as well, and hence, $(1+\lambda B)^{-1}$ is densely defined. Again, (4.1) implies that $(1+\lambda B)^{-1}$ is bounded with norm less than or equal to 1 . Thus, its closure is an element in $L(G)$ with the same norm.

For $\phi \in \operatorname{dom}(B)$ we obtain

$$
\overline{(1+\lambda B)^{-1}} \phi-\phi=-\overline{(1+\lambda B)^{-1}} \lambda B \phi \rightarrow 0 \quad(\lambda \rightarrow 0)
$$

and since $\operatorname{dom}(B)$ is dense and the family $\left(\overline{(1+\lambda B)^{-1}}\right)_{\lambda>0}$ is bounded, we infer that (4.3) holds for each $\phi \in G$.

We now prove the closability of $B$. For doing so, let $\left(\phi_{n}\right)_{n \in \mathbb{N}}$ in $\operatorname{dom}(B)$ with $\phi_{n} \rightarrow 0$ and $B \phi_{n} \rightarrow y$ for some $y \in G$ as $n \rightarrow \infty$. Thus, we infer for each $\lambda>0$

$$
\begin{aligned}
\overline{(1+\lambda B)^{-1}} y & =\lim _{n \rightarrow \infty} \overline{(1+\lambda B)^{-1}} B \phi_{n} \\
& =\frac{1}{\lambda} \lim _{n \rightarrow \infty}\left(\phi_{n}-\overline{(1+\lambda B)^{-1}} \phi_{n}\right) \\
& =0,
\end{aligned}
$$

and thus, letting $\lambda$ tend to $0,(4.3)$ yields $y=0$, proving that $B$ is closable. Noting that (4.1) and (4.2) yield that $B^{-1}$ is a densely defined bounded linear operator with norm less than or equal to $\frac{1}{c}$, the assertion follows with $\overline{B^{-1}}=\bar{B}^{-1}$.

The crucial part of the proof of Theorem 4.2 is to show that $\mathcal{B}$ has dense range. For this, we will employ the following lemma.

Lemma 4.4 Let $H$ be a Hilbert space, $D: \operatorname{dom}(D) \subseteq H \rightarrow H, C: \operatorname{dom}(C) \subseteq H \rightarrow H$ closed. Assume that $\operatorname{dom}(D) \cap \operatorname{dom}(C) \subseteq H$ is dense. Furthermore, let $\left(T_{n}\right)_{n}$ in $L(H)$ be such that $T_{n} \rightarrow 1$ in the strong operator topology. Moreover, assume that $T_{n}[\operatorname{dom}(D)] \subseteq$ $\operatorname{dom}(D)$ and $\operatorname{ran}\left(T_{n}\right) \subseteq \operatorname{dom}(C)$ for each $n \in \mathbb{N}$, as well as

$$
\begin{aligned}
& {\left[T_{n}, C\right],\left[T_{n}, D\right] \text { bounded for all } n \in \mathbb{N} \text {, }} \\
& \overline{\left[T_{n}, C\right]}, \overline{\left[T_{n}, D\right]} \rightarrow 0(n \rightarrow \infty),
\end{aligned}
$$

where the convergence holds in the strong operator topology. Then $(C+D)^{*}=\bar{C}^{*}+D^{*}$.

Proof. Note that $(C+D)^{*} \supseteq \overline{C^{*}+D^{*}}$ is clear. So, let $\phi \in \operatorname{dom}\left((C+D)^{*}\right)$ and for $n \in \mathbb{N}$ we define $\phi_{n}:=T_{n}^{*} \phi$. At first we show that $\phi_{n} \in \operatorname{dom}\left((C+D)^{*}\right)$. For this, let $\eta \in \operatorname{dom}(C+D)$. We compute

$$
\begin{aligned}
\left\langle(C+D) \eta, \phi_{n}\right\rangle & =\left\langle(C+D) \eta, T_{n}^{*} \phi\right\rangle \\
& =\left\langle T_{n}(C+D) \eta, \phi\right\rangle \\
& =\left\langle(C+D) T_{n} \eta, \phi\right\rangle+\left\langle\left[T_{n}, C\right] \eta, \phi\right\rangle+\left\langle\left[T_{n}, D\right] \eta, \phi\right\rangle \\
& =\left\langle\eta, T_{n}^{*}(C+D)^{*} \phi\right\rangle+\left\langle\eta,\left[T_{n}, C\right]^{*} \phi\right\rangle+\left\langle\eta,\left[T_{n}, D\right]^{*} \phi\right\rangle,
\end{aligned}
$$


which shows that $(C+D)^{*} \phi_{n}=T_{n}^{*}(C+D)^{*} \phi+\left[T_{n}, C\right]^{*} \phi+\left[T_{n}, D\right]^{*} \phi$. Next, note that $C T_{n} \in L(H)$ by the closed graph theorem and $\overline{\left[T_{n}, C\right]} \in L(H)$ by assumption. Hence, $\overline{T_{n} C}=\overline{\left[T_{n}, C\right]}+C T_{n} \in L(H)$ as well and thus, we deduce that ${\overline{T_{n} C^{*}}}^{*} C^{*} T_{n}^{*} \in L(H)$. In particular, we infer that $T_{n}^{*}$ maps into $\operatorname{dom}\left(C^{*}\right)$. Hence, $\phi_{n} \in \operatorname{dom}\left(C^{*}\right)$. Furthermore, for $\eta \in \operatorname{dom}(D)$, we have that $T_{m} \eta \in \operatorname{dom}(D) \cap \operatorname{dom}(C)$ by assumption. Moreover, we have

$$
T_{m} \eta \rightarrow \eta, \text { and } D T_{m} \eta=\left[D, T_{m}\right] \eta+T_{m} D \eta \rightarrow D \eta \quad(m \rightarrow \infty) .
$$

Thus, $\operatorname{dom}(C+D)$ is dense in $\operatorname{dom}(D)$ with respect to the graph norm of $D$. Altogether, we compute for all $\eta \in \operatorname{dom}(C+D)$

$$
\begin{aligned}
\left\langle D \eta, \phi_{n}\right\rangle & =\left\langle(C+D) \eta, \phi_{n}\right\rangle-\left\langle C \eta, \phi_{n}\right\rangle \\
& =\left\langle\eta,(C+D)^{*} \phi_{n}\right\rangle-\left\langle\eta, C^{*} \phi_{n}\right\rangle,
\end{aligned}
$$

which proves that $\phi_{n} \in \operatorname{dom}\left(D^{*}\right)$ and $D^{*} \phi_{n}=(C+D)^{*} \phi_{n}-C^{*} \phi_{n}$. Hence,

$$
T_{n}^{*}(C+D)^{*} \phi+\left[T_{n}, C\right]^{*} \phi+\left[T_{n}, D\right]^{*} \phi=(C+D)^{*} \phi_{n}=\left(D^{*}+C^{*}\right) \phi_{n} .
$$

Next, we may let $n \rightarrow \infty$ in the latter equality and obtain the assertion.

Proposition 4.5 Impose Assumption 4.1. Then $\operatorname{dom}\left(\partial_{0, \mu}\right)$ is a core for $\left(\partial_{0, \mu} \mathcal{M}\right)^{*}$.

Proof. It suffices to observe that $\operatorname{dom}\left(\partial_{0, \mu}\right)=\operatorname{dom}\left(\partial_{0, \mu}^{*}\right)$ and $\left(\partial_{0, \mu} \mathcal{M}\right)^{*}=\overline{\mathcal{M}^{*} \partial_{0, \mu}^{*}}$.

Proof of Theorem 4.2. Let $\mu \geq \nu$. First of all note that $\mathcal{B}$ is densely defined, since $\dot{C}_{\infty}(\mathbb{R} ; \operatorname{dom}(A)) \subseteq \operatorname{dom}(\mathcal{B})$. Moreover, note that

$$
\Re\langle\mathcal{B} \phi, \phi\rangle_{L_{\mu}^{2}} \geq c\langle\phi, \phi\rangle_{L_{\mu}^{2}}
$$

by Assumption 4.1. Next, since $\mathcal{B}$ is densely defined, we can use [2, Theorem 4.2.5], to deduce that $\mathcal{B}$ is closable. Note that inequality (4.4) remains true for $\phi \in \operatorname{dom}(\overline{\mathcal{B}})$.

We apply Lemma 4.3 to the operator $\overline{\mathcal{B}}$. For this, we compute the adjoint of $\mathcal{B}$. With the setting $C:=\partial_{0, \mu} \mathcal{M}_{\mu}+\mathcal{N}_{\mu}, D:=A$ and $T_{n}:=\left(1+(1 / n) \partial_{0, \mu}\right)^{-1}$, we employ Lemma 4.4. We check the hypothesis of Lemma 4.4 next. First of all, note that $T_{n}$ is well-defined with $\left\|T_{n}\right\| \leq 1$ and that $T_{n} \rightarrow 1$ in the strong operator topology. Clearly, $T_{n}$ leaves $\operatorname{dom}(D)$ invariant and attains values in $\operatorname{dom}(C)$. Moreover, both the operators

$$
T_{n} C \subseteq \partial_{0, \mu} T_{n} \mathcal{M}_{\mu}+T_{n} \mathcal{N}_{\mu} \text { and } C T_{n}=\left(\partial_{0, \mu} \mathcal{M}_{\mu}+\mathcal{N}_{\mu}\right) T_{n}=\left(\mathcal{M}_{\mu}^{\prime}+\mathcal{M}_{\mu} \partial_{0, \mu}+\mathcal{N}_{\mu}\right) T_{n}
$$

are densely defined and bounded. Thus, so is

$$
\left[T_{n}, C\right]=\left[T_{n}, \mathcal{N}_{\mu}\right]+\frac{1}{n} \partial_{0, \mu} T_{n} \mathcal{M}_{\mu}^{\prime} T_{n}
$$

It is not difficult to see that $\overline{\left[T_{n}, C\right]} \rightarrow 0$ as $n \rightarrow \infty$. Observe that

$$
\left[T_{n}, D\right] \subseteq 0 .
$$


So that $\overline{\left[T_{n}, D\right]}=0 \rightarrow 0$ as $n \rightarrow \infty$. Thus, by Lemma 4.4, we infer

$$
\mathcal{B}^{*}=(C+D)^{*}=\overline{C^{*}+D^{*}}=\overline{\left(\partial_{0, \mu} \mathcal{M}_{\mu}+\mathcal{N}_{\mu}\right)^{*}+A^{*}} .
$$

By the boundedness of $\mathcal{N}_{\mu}$, we deduce that $\left(\partial_{0, \mu} \mathcal{M}_{\mu}+\mathcal{N}_{\mu}\right)^{*}=\left(\partial_{0, \mu} \mathcal{M}_{\mu}\right)^{*}+\mathcal{N}_{\mu}^{*}$. Thus, by Proposition 4.5, $\operatorname{dom}\left(\partial_{0, \mu}\right)$ is an operator core for $C^{*}$. For $\phi \in \operatorname{dom}\left(\partial_{0, \mu}\right) \subseteq \operatorname{dom}(C)$ we compute

$$
\Re\left\langle C^{*} \phi, \phi\right\rangle_{\mu}=\Re\langle\phi, C \phi\rangle_{\mu} \geq c\langle\phi, \phi\rangle .
$$

Thus, $\Re\left\langle C^{*} \phi, \phi\right\rangle \geq c\langle\phi, \phi\rangle$ for all $\phi \in \operatorname{dom}\left(C^{*}\right)$. Moreover, since $A$ is densely defined and m-accretive, $A^{*}$ is accretive, as well, see [10]. Thus, altogether $\Re\left\langle\mathcal{B}^{*} \phi, \phi\right\rangle \geq c\langle\phi, \phi\rangle$. Therefore, Lemma 4.3 implies that $\mathcal{B}$ is continuously invertible and has dense range. In particular, we obtain $\mathcal{B}^{-1}$ is densely defined and has operator norm bounded by $1 / c$ so that $\mathcal{B}^{-1}$ is evolutionary at $\nu$.

For $\psi \in \operatorname{dom}\left(\mathcal{B}^{-1}\right), \phi:=\mathcal{B}^{-1} \psi$ we furthermore realize that the inequality

$$
\Re\left\langle\mathcal{B} \phi, Q_{t} \phi\right\rangle \geq c\left\langle Q_{t} \phi, Q_{t} \phi\right\rangle
$$

implies

$$
\left\|Q_{t} \mathcal{B}^{-1} \psi\right\| \leq \frac{1}{c}\left\|Q_{t} \psi\right\|
$$

which by Proposition 3.3 (iii) is sufficient for causality of $\overline{\mathcal{B}}^{-1}$.

Next, we slightly rephrase the main result of [17]. There, a well-posedness result for nonautonomous differential inclusions is stated, where the operator $A$ is replaced by a maximal monotone relation on a Hilbert space $H$ (for an introduction to maximal monotone relations on Hilbert spaces we refer to the monograph [3]). As a trade-off, we need to restrict the class of admissible operators $\mathcal{M}$ and $\mathcal{N}$ :

Theorem 4.6 ( [17, Theorem 3.4]) Let $H$ be a separable Hilbert space, let $M, N: \mathbb{R} \rightarrow$ $L(H)$ be strongly measurable and bounded mappings. Assume that $M(t)$ is selfadjoint for all $t \in \mathbb{R}, M$ Lipschitz continuous, $A \subseteq H \oplus H$ a maximal monotone relation with $(0,0) \in A$. Moreover, assume that $K:=\operatorname{ker}(M(t))=\operatorname{ker}(M(0))$ for all $t \in \mathbb{R}$ and that there exists $c>0$ such that for all $t \in \mathbb{R}$

$$
\langle M(t) \phi, \phi\rangle \geq c\langle\phi, \phi\rangle, \Re\langle N(t) \psi, \psi\rangle \geq c\langle\psi, \psi\rangle
$$

for all $\phi \in K$ and $\psi \in K^{\perp}$.

Then there exists $\nu>0, C \geq 0$ such that for all $\mu \geq \nu$

$$
\mathcal{S}_{\mu}:={\overline{\left(\partial_{0, \mu} \mathcal{M}+\mathcal{N}+A\right)}}^{-1}: L_{\nu}^{2}(\mathbb{R} ; H) \rightarrow L_{\nu}^{2}(\mathbb{R} ; H)
$$

is Lipschitz continuous with $\left\|\mathcal{S}_{\mu}\right\|_{\text {Lip }} \leq C$, causal and independent of $\mu$, where $\mathcal{M}, \mathcal{N}$ denote the abstract multiplication operators given by $(\mathcal{M} \phi)(t)=M(t) \phi(t)$ and $(\mathcal{N} \phi)(t)=$ $N(t) \phi(t)$, respectively. In particular, $\left.\mathcal{S}_{\mu}\right|_{C_{\infty}(\mathbb{R} ; H)}$ is densely defined, causal and evolutionary at $\nu$.

Although the latter theorem is a direct analogue of Theorem 4.2 in the nonlinear setting, its proof is completely different and rests on perturbation results for maximal monotone relations. As the proof is quite long and technical, we omit it here and refer to [17] instead. 


\section{Stochastic evolutionary equations}

Similar to the approach outlined in [15], we present the solution theory for stochastic partial differential equations based on Theorem 3.6. For this we first need to establish a suitable functional analytic formulation for the stochastic integral. In contrast to [15], where the authors focused on the case of Hilbert space valued Wiener processes, we shall favor a more axiomatic approach here. Indeed, this gives us more freedom for the choice of the stochastic processes in the integral. For this, we will introduce a class of 'admissible' processes and corresponding stochastic integrals. We mainly follow the rationale presented in [9].

\subsection{An abstract description of stochastic integration}

Throughout, we denote by $(\Omega, \Sigma, \mathbb{P})$ a probability space. Moreover, we fix a filtration $\mathcal{F}:=$ $\left(\Sigma_{t}\right)_{t \in \mathbb{R}}$, i.e. a family of sub- $\sigma$-algebras of $\Sigma$ satisfying

$$
\Sigma_{s} \subseteq \Sigma_{t} \quad(s \leq t)
$$

Moreover, we fix separable Hilbert spaces $G, H$ and a subspace $L \subseteq L(G, H)$ equipped with a Banach norm $\|\cdot\|_{L}$ such that

$$
\left(L,\|\cdot\|_{L}\right) \hookrightarrow(L(G, H),\|\cdot\|) .
$$

Definition 5.1 We collect some notions, which are needed in the following.

(a) We consider the following collection of sets

$$
\left.\{] s, t] \times A ; s, t \in \mathbb{R}, s<t, A \in \Sigma_{s}\right\} \subseteq \mathcal{P}(\mathbb{R} \times \Omega) .
$$

The $\sigma$-algebra generated by those sets is denoted by $\mathcal{B}_{\mathcal{F}}$ and is called the $\sigma$-algebra of $\mathcal{F}$-predictable sets.

(b) A mapping $X: \mathbb{R} \times \Omega \rightarrow Z$, where $Z$ is a Banach space, is called a stochastic process, if for each $t \in \mathbb{R}$ the mapping $X_{t}=X(t, \cdot): \Omega \rightarrow Z$ is measurable.

(c) A stochastic process $X: \mathbb{R} \times \Omega \rightarrow Z$ is called $\mathcal{F}$-adapted, if $X_{t}$ is $\Sigma_{t}$-measurable for each $t \in \mathbb{R}$. $X$ is called $\mathcal{F}$-predictable, if $X$ is $\mathcal{B}_{\mathcal{F}}$-measurable. For $\nu \geq 0$ we define

$$
L_{\nu, \operatorname{pr}}^{2}\left(\mathbb{R} ; L^{2}(\mathbb{P} ; Z)\right):=\left\{X \in L_{\nu}^{2}\left(\mathbb{R} ; L^{2}(\mathbb{P} ; Z)\right) ; X \text { predictable }\right\}
$$

which is a closed subspace of $L_{\nu}^{2}\left(\mathbb{R} ; L^{2}(\mathbb{P} ; Z)\right)$.

We now fix a stochastic process $X$ attaining values in $G$. The goal is now to define stochastic integration with respect to this process $X$. The integrands are suitable stochastic processes attaining values in $L$ and the integral should be an element in $H^{\Omega}$.

We start by defining

$$
I^{X}\left(T \chi_{] s, t] \times A}\right):=\int_{\mathbb{R}} T \chi_{] s, t] \times A} \mathrm{~d} X:=\left(\omega \mapsto \chi_{A}(\omega) T\left(X_{t}(\omega)-X_{s}(\omega)\right) .\right.
$$


where $s<t, A \in \Sigma_{s}$ and $T \in L$. Clearly, this integral operator $I^{X}$ can be extend to a linear operator on simple $\mathcal{F}$-predictable processes $Y: \mathbb{R} \times \Omega \rightarrow L$. We denote this linear extension again by $I^{X}$.

Moreover, if $X_{t} \in L^{2}(\mathbb{P} ; G)$ for each $t \in \mathbb{R}$ we immediately get that $I^{X}$ attains values in $L^{2}(\mathbb{P} ; H)$. The main idea is now to extend this integral operator to a broader class of processes. For doing so, we need to restrict to a certain class of processes $X$.

Definition 5.2 Let $X: \mathbb{R} \times \Omega \rightarrow G$ be such that $X_{t} \in L^{2}(\mathbb{P} ; G)$ for each $t \in \mathbb{R}$. We call $X$ an $L^{2}$-primitive, if there exists a measure $\alpha: \mathcal{B}_{\mathcal{F}} \rightarrow[0, \infty]$ and $C \geq 0$ such that

$$
\left|I^{X}\left(\sum_{i=0}^{n} T_{i} \chi_{] s_{i}, t_{i}\right] \times A_{i}}\right)\right|_{L^{2}(\mathbb{P} ; H)} \leq C\left|\sum_{i=0}^{n} T_{i} \chi_{] s_{i}, t_{i}\right] \times A_{i}}\right|_{L^{2}(\alpha ; L)},
$$

where $n \in \mathbb{N}, s_{i}<t_{i}, A_{i} \in \Sigma_{s_{i}}$ and $T_{i} \in L, i \in\{0, \ldots, n\}$.

In this case, $\alpha$ is called a dominating measure for $X$. We denote by $I^{X, \alpha}$ the unique extension of $I^{X}$ to a bounded linear operator

$$
I^{X, \alpha}: L^{2}(\alpha ; L) \rightarrow L^{2}(\mathbb{P} ; H)
$$

and call it the stochastic integral with respect to $X$ on $L^{2}(\alpha ; L)$. For $Y \in L^{2}(\alpha ; L)$ we also write

$$
\int_{\mathbb{R}} Y \mathrm{~d} X:=I^{X, \alpha}(Y)
$$

Remark 5.3 We note that in the latter definition the measure $\alpha$ may not be uniquely determined. Thus, the latter definition allows for the extension of the stochastic integral in various spaces. It is clear, however, that for two dominating measures $\alpha_{1}, \alpha_{2}$ we have that the two extension $I^{X, \alpha_{1}}$ and $I^{X, \alpha_{2}}$ coincide on the intersection $L^{2}\left(\alpha_{1} ; L\right) \cap L^{2}\left(\alpha_{2} ; L\right)$.

Example 5.4. By [9, Section 2.6] the following processes are $L^{2}$-primitives.

(a) Let $X$ satisfy $X_{t} \in L^{2}\left(\Omega, \Sigma_{t}, \mathbb{P} ; G\right)$ for each $t \in \mathbb{R}$ (i.e., $X$ is $\mathcal{F}$-adapted) and assume that

$$
\mathbb{R} \ni t \mapsto\left|X_{t}\right|_{L^{2}(\mathbb{P} ; G)}
$$

is right continuous. Moreover, we assume that $X$ has independent and centered increments, i.e., for each $s, t \in \mathbb{R}$ with $s<t$ and each $x \in G$ we have that

$$
\omega \mapsto\left\langle X_{t}(\omega)-X_{s}(\omega), x\right\rangle_{G}
$$

is independent of $\Sigma_{s}$ and

$$
\int_{\Omega}\left(X_{t}(\omega)-X_{s}(\omega)\right) \mathrm{d} \mathbb{P}(\omega)=0 .
$$

Then $X$ is an $L^{2}$-primitive with dominating measure $\alpha=\mu \otimes \mathbb{P}$, where $\mu$ is the Stieltjes measure associated with the function

$$
t \mapsto\left|X_{t}\right|_{L^{2}(\mathbb{P} ; G)}
$$

In particular, the Hilbert space valued Wiener process $W$ is an $L^{2}$-primitive, if we choose $\Sigma_{t}:=\sigma\left(W_{s} ; s \leq t\right)$. 
(b) More generally, if $X: \mathbb{R} \times \Omega \rightarrow G$ is an $\mathcal{F}$-martingale, such that $X_{t} \in L^{2}\left(\Omega, \Sigma_{t}, \mathbb{P} ; G\right)$ for each $t \in \mathbb{R}$ and

$$
t \mapsto\left|X_{t}\right|_{L^{2}(\mathbb{P} ; G)}
$$

is right-continuous, then $X$ is an $L^{2}$-primitive with dominating measure $\alpha=d_{|X|_{G}^{2}}$, the Doleans-measure of the submartingale $|X|_{G}^{2}$ (see e.g. [9, Section 1.20] or [5]).

Our next goal is to introduce a primitive of an $L$-valued process $Y$ with respect to a $G$ valued process $X$.

Proposition 5.5 Let $X: \mathbb{R} \times \Omega \rightarrow G$ be an $L^{2}$-primitive with dominating measure $\alpha$. Moreover, for $\nu>0$ we define the space $L_{\nu}^{2}(\alpha ; L)$ as the space of $L$-valued, $\mathcal{F}$-predictable processes $Y$ satisfying

$$
\int_{\mathbb{R} \times \Omega}|Y(t, \omega)|_{L}^{2} \exp (-2 \nu t) \mathrm{d} \alpha(t, \omega)<\infty .
$$

For $\mu>0$ we consider the operator

$$
\mathcal{I}^{X, \alpha}: S(\alpha ; L) \subseteq \bigcap_{\nu \geq \mu} L_{\nu}^{2}(\alpha ; L) \rightarrow \bigcap_{\nu \geq \mu} L_{\nu}^{2}\left(\mathbb{R} ; L^{2}(\mathbb{P} ; H)\right)
$$

given by

$$
\mathcal{I}^{X, \alpha}(Y):=\left(t \mapsto I^{X}\left(\chi_{\mathbb{R}_{\leq t}} Y\right)\right),
$$

where $S(\alpha ; L)$ denotes the space of simple $L$-valued, predictable processes.

Then $\mathcal{I}^{X, \alpha}$ is evolutionary at $\mu$ and densely defined. More precisely, there exists a constant $C \geq 0$ such that

$$
\left\|\mathcal{I}_{\nu}^{X, \alpha}\right\| \leq \frac{C}{\sqrt{2 \nu}} \quad(\nu \geq \mu)
$$

Moreover, $\mathcal{I}_{\nu}^{X, \alpha}$ is causal and $\mathcal{I}_{\nu}^{X, \alpha}$ and $\mathcal{I}_{\widetilde{\nu}}^{X, \alpha}$ coincide on the intersection $L_{\nu}^{2}(\alpha ; L) \cap L_{\widetilde{\nu}}^{2}(\alpha ; L)$ for each $\nu, \widetilde{\nu} \geq \mu$.

Proof. Let $Y \in S(\alpha ; L)$. First we note that $\chi_{\mathbb{R}_{\leq t}} Y \in S(\alpha ; L)$ for each $t \in \mathbb{R}$. Let now $\nu \geq \mu$. We estimate

$$
\begin{aligned}
& \int_{\mathbb{R}}\left|\mathcal{I}^{X, \alpha}(Y)(t)\right|_{L^{2}(\mathbb{P} ; H)}^{2} \exp (-2 \nu t) \mathrm{d} t \\
& \leq C^{2} \int_{\mathbb{R}} \int_{\mathbb{R} \times \Omega}\left|\chi_{\mathbb{R}_{\leq t}}(s) Y(s, \omega)\right|_{L}^{2} \mathrm{~d} \alpha(s, \omega) \exp (-2 \nu t) \mathrm{d} t \\
& =C^{2} \int_{\mathbb{R} \times \Omega} \int_{\mathbb{R}} \chi_{\mathbb{R}_{\geq s}}(t) \exp (-2 \nu t) \mathrm{d} t|Y(s, \omega)|_{L}^{2} \mathrm{~d} \alpha(s, \omega) \\
& =\frac{C^{2}}{2 \nu}|Y|_{L_{\nu}^{2}(\alpha ; L)}^{2},
\end{aligned}
$$


which shows that $\mathcal{I}^{X, \alpha}$ is evolutionary at $\mu$ and that the norm estimate holds. The causality and the independence on the parameter $\nu$ follows from Lemma 3.5 and Lemma 3.4.

Lemma 5.6 Let $X: \mathbb{R} \times \Omega \rightarrow G$ be an $L^{2}$-primitive with dominating measure $\alpha$ and assume that

$$
\mathbb{R} \ni t \mapsto X_{t}(\omega)
$$

is weakly left continuous. Moreover, we assume that $X$ is $\mathcal{F}$-adapted. Then for $\nu>0$ and $Y \in L_{\nu}^{2}(\alpha ; L)$ we have that

$$
\mathcal{I}^{X, \alpha}(Y) \in L_{\nu, \mathrm{pr}}^{2}\left(\mathbb{R} ; L^{2}(\mathbb{P} ; H)\right) .
$$

Consequently,

$$
\mathcal{I}^{X, \alpha}: L_{\nu}^{2}(\alpha ; L) \rightarrow L_{\nu, \mathrm{pr}}^{2}\left(\mathbb{R} ; L^{2}(\mathbb{P} ; H)\right)
$$

is a bounded linear operator.

Pro of. It suffices to prove that $\mathcal{I}^{X, \alpha}(Y)$ is predictable. Due to linearity and continuity it

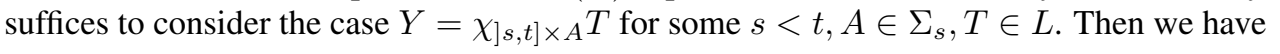

$$
\mathcal{I}^{X, \alpha}(Y)(\tau, \omega)=\chi_{] s, t] \times A}(\tau, \omega) T\left(X_{\tau}(\omega)-X_{s}(\omega)\right)+\chi_{] t, \infty] \times A}(\tau, \omega) T\left(X_{t}(\omega)-X_{s}(\omega)\right)
$$

for each $\tau \in \mathbb{R}, \omega \in \Omega$. Note that $\mathcal{I}^{X, \alpha}(Y)$ is $\mathcal{F}$-adapted and that $\tau \mapsto \mathcal{I}^{X, \alpha}(Y)(\tau, \omega)$ is weakly left continuous for each $\omega \in \Omega$. Thus, by [4, Proposition 3.7] it is $\mathcal{F}$-predictable.

\subsection{Solution theory for abstract stochastic evolutionary equations}

In the previous section, we have focused on the stochastic part of the evolutionary equation with stochastic perturbation. We are now in the position to combine the results of the previous sections in order to provide the desired solution theory. First of all, we state the main assumptions of this section.

Assumption 5.7 Let $G, H$ be two separable Hilbert spaces and $L \subseteq L(G, H)$ a subspace equipped with a Banach norm, such that $L \hookrightarrow L(G, H)$. Moreover, let $(\Omega, \Sigma, \mathbb{P})$ be a probability space and $\mathcal{F}=\left(\Sigma_{t}\right)_{t \in \mathbb{R}}$ a filtration. We fix an $\mathcal{F}$-adapted process $X: \mathbb{R} \times \Omega \rightarrow G$, which is an $L^{2}$-primitive with dominating measure $\alpha$ and we assume that

$$
t \mapsto X_{t}(\omega)
$$

is weakly left continuous for each $\omega \in \Omega$. Moreover, we assume that $L_{0, p r}^{2}\left(\mathbb{R} ; L^{2}(\mathbb{P})\right) \hookrightarrow$ $L^{2}(\alpha)$.

We first recall the central observation of [15] in a slightly different way.

Theorem 5.8 ( [15, Theorem 3.4]) Let $\nu>0$ and $M \in L\left(L_{\nu}^{2}(\mathbb{R} ; H)\right)$ be causal. Then the canonical extension of $M$ to $L_{\nu}^{2}\left(\mathbb{R} ; L^{2}(\mathbb{P} ; H)\right)$ given by

$$
(M u)(t, \omega):=M(u(\cdot, \omega))(t) \quad(t \in \mathbb{R}, \omega \in \Omega)
$$

leaves the space of predictable processes invariant, that is,

$$
M\left[L_{\nu, \operatorname{pr}}^{2}\left(\mathbb{R} ; L^{2}(\mathbb{P} ; H)\right)\right] \subseteq L_{\nu, \operatorname{pr}}^{2}\left(\mathbb{R} ; L^{2}(\mathbb{P} ; H)\right) .
$$


Before we can come to our main well-posedness result, we need the following lemma.

Lemma 5.9 Let $\mu>0, \sigma: H \rightarrow L$ be Lipschitz continuous, $\sigma(0)=0$, and define

$$
\widetilde{\sigma}: \bigcap_{\nu \geq \mu} L_{\nu}^{2}\left(\mathbb{R} ; L^{2}(\mathbb{P} ; H)\right) \rightarrow \bigcap_{\nu \geq \mu} L_{\nu}^{2}\left(\mathbb{R} ; L^{2}(\mathbb{P} ; L)\right)
$$

by

$$
(\widetilde{\sigma} u)(t, \omega):=\sigma(u(t, \omega)) .
$$

Then $\widetilde{\sigma}$ is evolutionary at $\mu, \widetilde{\sigma}_{\nu}$ is causal and does not depend on the parameter $\nu$. Moreover, for $\nu \geq \mu$ we have that the restriction

$$
\widetilde{\sigma}_{\nu}: L_{\nu, \operatorname{pr}}^{2}\left(\mathbb{R} ; L^{2}(\mathbb{P} ; H)\right) \rightarrow L_{\nu}^{2}(\alpha ; L)
$$

is well-defined and Lipschitz continuous, where the smallest Lipschitz constant can be chosen independent of $\nu$.

Pro of. It is obvious, that $\widetilde{\sigma}$ is again Lipschitz continuous with the same Lipschitz constant as $\sigma$. Hence, it is evolutionary at $\mu$. The causality and independence of the parameter follows by Lemma 3.4 and Lemma 3.5. We now come to the second assertion. We first show that $\tilde{\sigma}(u)$ is predictable if $u$ is predictable. Note that by continuity it suffices to prove this for $u=\sum_{i=1}^{n} \chi_{\left.s_{i}, t_{i}\right] \times A_{i}} x_{i}$ for $s_{i}<t_{i}, A_{i} \in \Sigma_{s_{i}}, x_{i} \in H, i \in\{1, \ldots, n\}$. We may assume without loss of generality that the intervals $\left.] s_{i}, t_{i}\right]$ are pairwise disjoint. Then

$$
\tilde{\sigma}(u)=\sum_{i=1}^{n} \chi_{] s_{i}, t_{i}\right] \times A_{i}} \sigma\left(x_{i}\right)
$$

and hence, $\widetilde{\sigma}(u)$ is predictable. From $L_{0, \mathrm{pr}}^{2}\left(\mathbb{R} ; L^{2}(\mathbb{P})\right) \hookrightarrow L^{2}(\alpha)$ we infer

$$
L_{\nu, \operatorname{pr}}^{2}\left(\mathbb{R} ; L^{2}(\mathbb{P} ; L)\right) \hookrightarrow L_{\nu}^{2}(\alpha ; L)
$$

for each $\nu \geq 0$ where the embedding constant is independent of $\nu$. Thus, as we have shown that

$$
\widetilde{\sigma}_{\nu}: L_{\nu, \mathrm{pr}}^{2}\left(\mathbb{R} ; L^{2}(\mathbb{P} ; H)\right) \rightarrow L_{\nu, \mathrm{pr}}^{2}\left(\mathbb{R} ; L^{2}(\mathbb{P} ; L)\right)
$$

is well-defined and Lipschitz continuous with a Lipschitz constant independent of $\nu$, the assertion follows.

Corollary 5.10 Let $\mu>0, \sigma: H \rightarrow L$ be Lipschitz continuous, $\sigma(0)=0$. Then

$$
\mathcal{I}^{X, \alpha} \circ \tilde{\sigma}: \bigcap_{\nu \geq \mu} L_{\nu, \operatorname{pr}}^{2}\left(\mathbb{R} ; L^{2}(\mathbb{P} ; H)\right) \subseteq \bigcap_{\nu \geq \mu} L_{\nu}^{2}\left(\mathbb{R} ; L^{2}(\mathbb{P} ; H)\right) \rightarrow \bigcap_{\nu \geq \mu} L_{\nu}^{2}\left(\mathbb{R} ; L^{2}(\mathbb{P} ; H)\right)
$$

is invariant evolutionary and $\left\|\mathcal{I}^{X, \alpha} \circ \widetilde{\sigma}\right\|_{\mathrm{ev}, \mathrm{Lip}}=0$.

Proof. By Proposition 5.5 and Lemma 5.9 we infer that $\mathcal{I}^{X, \alpha} \circ \widetilde{\sigma}$ is evolutionary with $\left\|\mathcal{I}^{X, \alpha} \circ \tilde{\sigma}\right\|_{\text {ev }, \text { Lip }}=0$. The invariance follows by Lemma 5.6 and Lemma 5.9. 
We are now in the position to formulate the abstract solution theory, which is based on Theorem 3.6.

Theorem 5.11 Impose Assumption 4.1 and let $\sigma: H \rightarrow L$ be Lipschitz continuous, $\sigma(0)=$ 0 . Then there exists $\nu>0$ such that for all $f \in L_{\nu, \mathrm{pr}}^{2}\left(\mathbb{R} ; L^{2}(\mathbb{P} ; H)\right)$ there exists a unique $u_{f} \in L_{\nu, \mathrm{pr}}^{2}\left(\mathbb{R} ; L^{2}(\mathbb{P} ; H)\right)$ such that

$$
\overline{\left(\partial_{0, \nu} \mathcal{M}+\mathcal{N}+A\right)} u_{f}=f+\mathcal{I}_{\nu}^{X, \alpha} \circ \widetilde{\sigma}\left(u_{f}\right) .
$$

The mapping $f \mapsto u_{f}$ is causal and does not depend on $\nu$ in the sense of Lemma 3.4.

Proof. We use Theorem 3.6. For this, we observe that $S:=\left(\partial_{0, \nu} \mathcal{M}+\mathcal{N}+A\right)^{-1}$ is evolutionary, causal and densely defined, by Theorem 4.2. Moreover, since $S$ is causal, we have that $S_{\mu}\left[\operatorname{dom}\left(F_{\mu}\right)\right] \subseteq \operatorname{dom}\left(F_{\mu}\right)$ for all $\mu \geq \nu$ with $F:=\mathcal{I}^{X, \alpha} \circ \widetilde{\sigma}$, by Theorem 5.8. Moreover, by Corollary 5.10, we deduce that $F$ is invariant evolutionary and $\|F\|_{\text {ev,Lip }}=0$. Thus Theorem 3.6 is applicable and we obtain the assertion.

Also in the non-linear setting, we obtain an analogous result with exactly the same proof, where we use Theorem 4.6 instead of Theorem 4.2.

Theorem 5.12 Let $M, N: \mathbb{R} \rightarrow L(H)$ weakly measurable and $M$ Lipschitz continuous. Let $A \subseteq H \otimes H$ maximal monotone with $(0,0) \in A$ and $\sigma: H \rightarrow L$ Lipschitz continuous, $\sigma(0)=0$. Moreover, assume that $K:=\operatorname{ker}(M(t))=\operatorname{ker}(M(0))$ for all $t \in \mathbb{R}$ and that there exists $c>0$ such that for all $t \in \mathbb{R}$

$$
\langle M(t) \phi, \phi\rangle \geq c\langle\phi, \phi\rangle, \Re\langle N(t) \psi, \psi\rangle \geq c\langle\psi, \psi\rangle
$$

for all $\phi \in K$ and $\psi \in K^{\perp}$. Then there exists $\nu>0$ such that for all $f \in L_{\nu, \mathrm{pr}}^{2}\left(\mathbb{R} ; L^{2}(\mathbb{P} ; H)\right)$ there exists a unique $u_{f} \in L_{\nu, \mathrm{pr}}^{2}\left(\mathbb{R} ; L^{2}(\mathbb{P} ; H)\right)$ such that

$$
\overline{\left(\partial_{0, \nu} \mathcal{M}+\mathcal{N}+A\right)} \ni\left(u_{f}, f+\mathcal{I}_{\nu}^{X, \alpha} \circ \widetilde{\sigma}\left(u_{f}\right)\right)
$$

The mapping $f \mapsto u_{f}$ is causal and does not depend on $\nu$ in the sense of Lemma 3.4.

\subsection{An abstract stochastic heat/wave equation}

In this section, we treat an abstract example of an equation of mixed type. For this let $H_{1}, H_{2}$ be separable Hilbert spaces, $C: \operatorname{dom}(C) \subseteq H_{1} \rightarrow H_{2}$ be closed and densely defined. We assume Assumption 5.7 with $H$ replaced by $H_{1}$. Let $a: \mathbb{R} \rightarrow L\left(H_{2}\right)$ be bounded and Lipschitz continuous satisfying $a(t)=a(t)^{*} \geq c$ for all $t \in \mathbb{R}$ and some $c>0$; we denote by $a(\mathrm{~m})$ the abstract multiplication operator realized as an operator from $L_{\nu}^{2}\left(\mathbb{R} ; H_{2}\right)$ to $L_{\nu}^{2}\left(\mathbb{R} ; H_{2}\right)$ for all $\nu>0$. Moreover, let $\sigma: H_{1} \rightarrow L$ be Lipschitz continuous with $\sigma(0)=0$ and $P=P^{*}=P^{2} \in L\left(H_{1}\right)$.

The problem we are about to study with regards to well-posedness issues reads as follows. Let $f \in L_{\nu, \mathrm{pr}}^{2}\left(\mathbb{R} ; L^{2}\left(\mathbb{P} ; H_{1}\right)\right)$ be given. Then (write $\partial_{0}$ for the time derivatve) consider the equation

$$
\partial_{0}^{2} P u+\partial_{0}(1-P) u+C^{*} a(\mathrm{~m}) C u=f+\partial_{0}\left(\mathcal{I}^{X, \alpha} \circ \tilde{\sigma}\right)(u) .
$$


Note that for the special cases $P=1_{H_{1}}$ and $P=0$, we recover the respective special cases of an abstract wave equation and an abstract heat equation.

In applications, for instance if $X$ is a Wiener process, the expression $\partial_{0} \mathcal{I}^{X, \alpha} \circ \widetilde{\sigma}(u)$ is often written as $\sigma(u(t)) \dot{W}(t)$. Then $\int_{0}^{t} \sigma(u(t)) \dot{W}(t) d t$ is interpreted as stochastic integral. Here, we employ the same rationale since $\partial_{0}^{-1}$ is integration (see Section 3) so that $\partial_{0}^{-1} \partial_{0} \mathcal{I}^{X, \alpha} \circ \widetilde{\sigma}=$ $\mathcal{I}^{X, \alpha} \circ \widetilde{\sigma}$.

In order to apply the solution theory outlined in the previous section, we shall reformulate (5.1). Denote $q:=\partial_{0}^{-1} a(\mathrm{~m}) C u$. Thus, (5.1) reads

$$
\begin{aligned}
\left(\partial_{0}\left(\begin{array}{cc}
P & 0 \\
0 & b(\mathrm{~m})
\end{array}\right)+\left(\begin{array}{cc}
(1-P) & 0 \\
0 & -b^{\prime}(\mathrm{m})
\end{array}\right)+\right. & \left.\left(\begin{array}{cc}
0 & C^{*} \\
-C & 0
\end{array}\right)\right)\left(\begin{array}{l}
u \\
q
\end{array}\right) \\
& =\left(\begin{array}{c}
\partial_{0}^{-1} f+\mathcal{I}^{X, \alpha} \circ \widetilde{\sigma}(u) \\
0
\end{array}\right),
\end{aligned}
$$

where $b(t):=a(t)^{-1}$ for all $t \in \mathbb{R}$ and $b^{\prime}$ is the weak derivative of $b$.

Identifying $x \in H_{1}$ with $x \oplus 0 \in H_{1} \oplus H_{2}$ we realize that Theorem 5.11 applies once we have shown that Assumption 4.1 is satisfied for the following setting

$$
\begin{aligned}
& H=H_{1} \oplus H_{2}, \mathcal{M}=\left(\begin{array}{cc}
P & 0 \\
0 & b(\mathrm{~m})
\end{array}\right), \mathcal{N}=\left(\begin{array}{cc}
(1-P) & 0 \\
0 & -b^{\prime}(\mathrm{m})
\end{array}\right), \\
& A=\left(\begin{array}{cc}
0 & C^{*} \\
-C & 0
\end{array}\right), \mathcal{M}^{\prime}=\left(\begin{array}{cc}
0 & 0 \\
0 & b^{\prime}(\mathrm{m})
\end{array}\right),
\end{aligned}
$$

and a suitably chosen $\nu>0$. The rest of this section is devoted to verify the conditions in Assumption 4.1.

Lemma 5.13 The operator $A$ is skew-selfadjoint. In particular, $\Re\langle A x, x\rangle=0$ for all $x \in \operatorname{dom}(A)=\operatorname{dom}\left(A^{*}\right)$ and $\operatorname{ran}(A \pm 1)=H$, so that $A$ is $m$-accretive.

Pro of. The claim follows once we realize that for densely defined operators $B_{1}, B_{2}$ acting in appropriate Hilbert space, we have $\left(\begin{array}{cc}0 & B_{1}^{*} \\ B_{2}^{*} & 0\end{array}\right)=\left(\begin{array}{cc}0 & B_{2} \\ B_{1} & 0\end{array}\right)^{*}$.

Lemma 5.14 For all $\nu \in \mathbb{R}$, we have

$$
\partial_{0, \nu} \mathcal{M} \subseteq \mathcal{M} \partial_{0, \nu}-\mathcal{M}^{\prime}
$$

Proof. Let $s, t \in \mathbb{R}$. Then we compute

$$
\begin{aligned}
\|b(t)-b(s)\| & =\left\|a(t)^{-1}-a(s)^{-1}\right\| \\
& =\left\|a(t)^{-1}(a(s)-a(t)) a(s)^{-1}\right\| \\
& \leq \frac{1}{c^{2}}\|a(s)-a(t)\| \leq \frac{1}{c^{2}}\|a\|_{\text {Lip }}|s-t| .
\end{aligned}
$$

Thus, $b$ is Lipschitz continuous and $H_{2}$ is separable, thus, $b \phi$ is weakly differentiable for all $\phi \in \stackrel{C}{C}_{\infty}\left(\mathbb{R} ; H_{2}\right)$ and

$$
(b \phi)^{\prime}=b^{\prime} \phi+b \phi^{\prime},
$$

where $b^{\prime}$ is the strong derivative defined by the derivative of $b^{\prime}(t) x:=(b(\cdot) x)^{\prime}(t)$ for almost all $t \in \mathbb{R}, x \in H_{2}$, see also [12, Lemma 2.1]. Since $P \partial_{0, \nu} \subseteq \partial_{0, \nu} P$, the claim follows. 
A particular observation in the latter proof is that $b$ is Lipschitz continuous and $\|b\|_{\text {Lip }} \leq$ $\frac{1}{c^{2}}\|a\|_{\text {Lip. }}$

Lemma 5.15 There exist $k, \nu>0$ such that for all $t \in \mathbb{R}$ and $\phi \in \stackrel{\circ}{C}_{\infty}(\mathbb{R} ; H)$ we have

$$
\Re\left\langle\left(\partial_{0, \nu} \mathcal{M}+\mathcal{N}\right) \phi, Q_{t} \phi\right\rangle_{L_{\nu}^{2}} \geq k\left\langle Q_{t} \phi, \phi\right\rangle_{L_{\nu}^{2}} .
$$

Pro of. Since $\mathcal{M}, \mathcal{M}^{\prime}, \mathcal{N}$ are diagonal block operator matrices, it suffices to restrict ourselves to test functions $\phi$ in $\stackrel{\circ}{C}_{\infty}\left(\mathbb{R} ; H_{1}\right)$ and $\stackrel{\circ}{C}_{\infty}\left(\mathbb{R} ; H_{2}\right)$. We can apply [12, Lemma 2.6] to $M_{0}(\mathrm{~m})=(1-P), M_{1}(\mathrm{~m})=P, A=0$ to obtain for all $t \in \mathbb{R}, \nu>0$ :

$$
\Re\left\langle\partial_{0, \nu} P \phi+(1-P) \phi, Q_{t} \phi\right\rangle_{L_{\nu}^{2}}=\frac{1}{2}\langle\phi(t), P \phi(t)\rangle_{H_{1}} e^{-2 \nu t}+\left\langle\nu P \phi+(1-P) \phi, Q_{t} \phi\right\rangle_{L_{\nu}^{2}} .
$$

Thus, for all $\phi \in \stackrel{\circ}{C}_{\infty}\left(\mathbb{R} ; H_{1}\right)$

$$
\Re\left\langle\partial_{0, \nu} P \phi+(1-P) \phi, Q_{t} \phi\right\rangle_{L_{\nu}^{2}} \geq \min \{\nu, 1\}\left\langle\phi, Q_{t} \phi\right\rangle_{L_{\nu}^{2}} .
$$

Next, again by [12, Lemma 2.6] this time applied to $M_{0}(\mathrm{~m})=b(\mathrm{~m}), M_{1}(\mathrm{~m})=-b^{\prime}(\mathrm{m})$, and $A=0$, we compute

$$
\begin{aligned}
\Re\left\langle\partial_{0, \nu} b(\mathrm{~m}) \phi-b^{\prime}\right. & \left.(\mathrm{m}) \phi, Q_{t} \phi\right\rangle_{L_{\nu}^{2}} \\
& =\frac{1}{2}\langle\phi(t), b(t) \phi(t)\rangle_{H_{1}} e^{-2 \nu t}+\left\langle\nu b(\mathrm{~m}) \phi-\frac{1}{2} b^{\prime}(\mathrm{m}) \phi, Q_{t} \phi\right\rangle_{L_{\nu}^{2}},
\end{aligned}
$$

where we used that $b^{\prime}(t)$ is selfadjoint as $b(s)$ is selfadjoint for all $s \in \mathbb{R}$. We observe that the non-negativity of $a$ implies the same for $b$; more precisely we get $b(t) \geq \frac{c}{\|a(t)\|^{2}} \geq$ $\frac{c}{\sup _{t \in \mathbb{R}}\|a(t)\|^{2}}=: c^{\prime}>0$. Moreover, we note that $\left\|b^{\prime}(\mathrm{m})\right\| \leq\|b\|_{\text {Lip }}$, by [12, Lemma 2.1]. Hence, $\left\|b^{\prime}(\mathrm{m})\right\| \leq \frac{1}{c^{2}}\|a\|_{\text {Lip. }}$. Thus, we deduce that

$$
\begin{aligned}
\Re\left\langle\partial_{0, \nu} b(\mathrm{~m}) \phi-b^{\prime}(\mathrm{m}) \phi, Q_{t} \phi\right\rangle_{L_{\nu}^{2}} & \geq\left\langle\nu b(\mathrm{~m}) \phi-\frac{1}{2} b^{\prime}(\mathrm{m}) \phi, Q_{t} \phi\right\rangle_{L_{\nu}^{2}} \\
& =\left\langle\left(\nu b(\mathrm{~m})-\frac{1}{2} b^{\prime}(\mathrm{m})\right) Q_{t} \phi, Q_{t} \phi\right\rangle_{L_{\nu}^{2}} \\
& \geq\left(\nu c^{\prime}-\frac{1}{c^{2}}\|a\|_{\text {Lip }}\right)\left\langle Q_{t} \phi, Q_{t} \phi\right\rangle_{L_{\nu}^{2}},
\end{aligned}
$$

which yields the assertion.

The Lemmas 5.13, 5.14, and 5.15 finally yield the applicability of Theorem 5.11, so that (5.2) is well-posed. In fact, we have the following result:

Theorem 5.16 There exists $\nu>0$ such that for all $f \in L_{\nu, \mathrm{pr}}^{2}\left(\mathbb{R} ; L^{2}\left(\mathbb{P} ; H_{1}\right)\right)$ we find a uniquely determined $u_{f} \in L_{\nu, \mathrm{pr}}^{2}\left(\mathbb{R} ; L^{2}\left(\mathbb{P} ; H_{1}\right)\right)$ and $q_{f} \in L_{\nu, \mathrm{pr}}^{2}\left(\mathbb{R} ; L^{2}\left(\mathbb{P} ; H_{2}\right)\right)$ such that

$$
\begin{aligned}
& \overline{\left(\partial_{0, \nu}\left(\begin{array}{cc}
P & 0 \\
0 & b(\mathrm{~m})
\end{array}\right)+\left(\begin{array}{cc}
1-P & 0 \\
0 & -b^{\prime}(\mathrm{m})
\end{array}\right)+\left(\begin{array}{cc}
0 & \operatorname{div} \\
\operatorname{grad} & 0
\end{array}\right)\right)}\left(\begin{array}{l}
u_{f} \\
q_{f}
\end{array}\right) \\
& =\left(\begin{array}{c}
\partial_{0, \nu}^{-1} f+\mathcal{I}^{X, \alpha} \circ \tilde{\sigma}\left(u_{f}\right) \\
0
\end{array}\right) .
\end{aligned}
$$

If $f=0$ on $(-\infty, t]$ for some $t \in \mathbb{R}$, then $u_{f}=0$ and $q_{f}=0$ on $(-\infty, t]$. 
Remark 5.17 Using the notion of extrapolation spaces, we can make sense of the expression $\sigma(u(t)) \dot{X}(t)$, which might seem to be quite formal at first glance. For this we define the spaces $L_{\nu, \mathrm{pr},-1}^{2}\left(\mathbb{R} ; L^{2}(\mathbb{P} ; H)\right)$ as the completion of $L_{\nu, \mathrm{pr}}^{2}\left(\mathbb{R} ; L^{2}(\mathbb{P} ; H)\right)$ with respect to the norm $\left|\partial_{0, \nu}^{-1} \cdot\right|_{L_{\nu}^{2}\left(\mathbb{R} ; L^{2}(\mathbb{P} ; H)\right)}$ and $H_{-1}(A)$ as the completion of $H$ with respect to $\left|(1+A)^{-1} \cdot\right|_{H}$. Now, fix $f$ and let $(u, q)$ solve (5.2). Then we have that

$$
\begin{aligned}
& \overline{\left(\partial_{0, \nu}\left(\begin{array}{cc}
P & 0 \\
0 & b(\mathrm{~m})
\end{array}\right)+\left(\begin{array}{cc}
(1-P) & 0 \\
0 & -b^{\prime}(\mathrm{m})
\end{array}\right)+\left(\begin{array}{cc}
0 & C^{*} \\
-C & 0
\end{array}\right)\right)}\left(\begin{array}{l}
u \\
q
\end{array}\right) \\
& =\left(\begin{array}{c}
\partial_{0}^{-1} f+\mathcal{I}^{X, \alpha} \circ \tilde{\sigma}(u) \\
0
\end{array}\right)
\end{aligned}
$$

as an equation in $L_{\nu, \mathrm{pr}}^{2}\left(\mathbb{R} ; L^{2}(\mathbb{P} ; H)\right)$ for some large enough $\nu>0$. Using the continuous extensions of $\partial_{0, \nu}$ and $A=\left(\begin{array}{cc}0 & C^{*} \\ -C & 0\end{array}\right)$ with values in $L_{\nu, \mathrm{pr},-1}^{2}\left(\mathbb{R} ; L^{2}(\mathbb{P} ; H)\right)$ and $L_{\nu, \operatorname{pr}}^{2}\left(\mathbb{R} ; L^{2}\left(\mathbb{P} ; H_{-1}(A)\right)\right)$, respectively, we deduce that the equation satisfied by $(u, q)$ can be written without the closure bar. Moreover, one can differentiate line by line to obtain

$$
\partial_{0, \nu}^{2} P u+\partial_{0, \nu}(1-P) u+C^{*} \partial_{0, \nu} q=f+\partial_{0} \mathcal{I}^{X, \alpha} \circ \widetilde{\sigma}(u)
$$

and

$$
a(\mathrm{~m})^{-1} \partial_{0, \nu} q=C u
$$

which in turn formally gives back the system one started out with.

We remark that the well-posedness of (5.2) is also covered by Theorem 5.12, as $\mathcal{M}$ and $\mathcal{N}$ are given as abstract multiplication operators. Hence, we could generalize (5.2) by replacing the operator $A$ by a maximal monotone relation. This allows for instance the treatment of certain hysteresis effects in the theory of plasticity (see [16, 17]) or Section 2.2 above.

Remark 5.18 We shall comment on the limitations of the approach at hand.

(a) First of all, the developed theory is a Hilbert space approach and so certain nonlinear equations as for instance the ones in [8] cannot be treated right away. In fact, the deterministic solution theory of evolutionary equations in the form discussed in this manuscript is developed for the Hilbert space case only, as of yet, that is. We shall on the other hand emphasise that our considerations do not need any assumptions on the shape of the underlying physical domain. For some results in the deterministic Banach space case we refer to [22].

(b) The non-autonomous equations discussed here are formulated in a way that the unbounded operator (relation) is independent of the time variable. Again, this is already visible in the deterministic case. A treatment of spdes viewing the spatial operator being timedependent focussing on explicit (stochastic) partial differential equations can be found in [6]. The unbounded operators are then considered to be of elliptic type. This provides a larger class of equations with elliptic and time-dependent spatial operator, which is accessible with [6]. On the other hand, that approach makes it difficult to handle the full Maxwell system, see Section 2.1. 


\section{References}

[1] H.-D. Alber. Materials with memory. Initial-boundary value problems for constitutive equations with internal variables., volume 1682 of Lecture Notes in Mathematics. Springer, Berlin, 1998.

[2] H. R. Beyer. Beyond partial differential equations, volume 1898 of Lecture Notes in Mathematics. Springer, Berlin, 2007. On linear and quasi-linear abstract hyperbolic evolution equations.

[3] H. Brezis. Operateurs maximaux monotones et semi-groupes de contractions dans les espaces de Hilbert: cours. Universite Paris VI et CNRS-ERA 215, 1971.

[4] G. DaPrato and J. Zabczyk. Stochastic Equations in Infinite Dimensions, volume 45 of Encyclopedia of Mathematics and its Applications. Cambridge University Press, 2008.

[5] C. Doleans. Existence du processus croissant naturel associate un potentiel de la classe (d). Zeitschrift für Wahrscheinlichkeitstheorie und Verwandte Gebiete, 9(4):309-314, 1968.

[6] W. Liu and M. Röckner Stochastic partial differential equations: an introduction. Springer, Cham, 2015.

[7] A. Kalauch, R. Picard, S. Siegmund, S. Trostorff, and M. Waurick. A Hilbert Space Perspective on Ordinary Differential Equations with Memory Term. Journal of Dynamics and Differential Equations, 26(2):369-399, 2014.

[8] N.V. Krylov. An analytic approach to SPDEs. Stochastic partial differential equations: six perspectives, 185-242, Math. Surveys Monogr., 64, Amer. Math. Soc., Providence, RI, 1999.

[9] M. Metivier and J. Pellaumail. Stochastic integration. Probability and Mathematical Statistics. New York etc.: Academic Press (A Subsidiary of Harcourt Brace Jovanovich, Publishers), 1980.

[10] R. S. Phillips. Dissipative operators and hyperbolic systems of partial differential equations. Trans. Amer. Math. Soc., 90:193-254, 1959.

[11] R. Picard. A structural observation for linear material laws in classical mathematical physics. Mathematical Methods in the Applied Sciences, 32:1768-1803, 2009.

[12] R. Picard, S. Trostorff, M. Waurick, and M. Wehowski. On Non-Autonomous Evolutionary Problems. Journal of Evolution Equations, 13:751-776, 2013.

[13] C. Prevot and M. Röckner. A Concise Course on Stochastic Partial Differential Equations. Springer, 2007.

[14] B. Rozowskii. Stochastic Evolution Systems, volume 35 of Mathematics and its Applications. Kluwer Academic Publishers Group, 1990.

[15] A. Süß and M. Waurick. A Solution Theory for a General Class of SPDEs. Stochastics and Partial Differential Equations: Analysis and Computations, 5(2):278-318, 2017.

[16] S. Trostorff. An alternative approach to well-posedness of a class of differential inclusions in Hilbert spaces. Nonlinear Anal., 75(15):5851-5865, 2012.

[17] S. Trostorff and M. Wehowski. Well-posedness of non-autonomous evolutionary inclusions. Nonlinear Anal., 101:47-65, 2014.

[18] J. B. Walsh. An Introduction to Stochastic Partial Differential Equations, volume 1180. Springer, 1986.

[19] M. Waurick. A note on causality in Banach spaces. Indagationes Mathematicae, 26(2):404-412, 2015.

[20] M. Waurick. On Non-Autonomous Integro-Differential-Algebraic Evolutionary Problems. Mathematical Methods in the Applied Sciences, 38(4):665-676, 2015.

[21] M. Waurick. On the continuous dependence on the coefficients of evolutionary equations. Habilitation thesis, Technische Universität Dresden, 2016. http://arxiv.org/abs/1606.07731.

[22] M. Wehowski. Well-posedness of degenerate nonlinear Cauchy problems in Hilbert spaces. $\mathrm{PhD}$ thesis, TU Dresden, 2015 